\title{
Design of Dual-Band Circular Microstrip Patch Antenna for L-Band Military Applications
}

\author{
Settapong Malisuwan, Noppadol Tiamnara, and Nattakit Suriyakrai
}

\begin{abstract}
The shorter wavelength of L-band compared to UHF permit users to adopt smaller and more cost effective antenna. A smaller size microstrip antenna is particularly attractive for military use due to greater mobility and ease of camouflage. This paper presents a dual-band circular patch microstrip antenna for L-band military applications. The proposed antenna produced a bandwidth of $1.62-1.7 \mathrm{GHz}$ and $2.74-2.8 \mathrm{GHz}$. The simulated results are calculated by using IE3D software and MATLAB programming. Further, for the future work, analogous to dielectric relaxation consideration of Cole-Cole diagrams as applied to dielectric materials, a "reactive relaxation concept" will be introduced to present the frequency-dependent characteristics of the circular microstrip antenna.
\end{abstract}

Index Terms-Microstrip, antenna, dual-band, L-band, military.

\section{INTRODUCTION}

L-band (1-2GHz) has been used in various services including radar, aeronautical telemetry, fixed links, mobile communications, and satellite services [1]. This includes the usage in Global Position System (GPS), satellite mobile phones like Iridium, communication systems for various terrains like Inmarsat, and WorldSpace satellite radio.

For satellite communications, when compared to the widely used C-band (4-8GHz) and Ku-band (12-18GHz), L-band is relatively low in frequency, which in turn means easier to process, less complicated systems, and less susceptible to severe weather conditions at lower costs. Although higher bandwidth is available in Ku-band and C-band suitable for broadband communications like video, L-band is more cost-effective for voice and low-speed data communications.

Unlike Ku- and C-band, L-band systems do not require strictly accurate antenna pointing. Due to its wider beam width, L-band terminals only need general pointing towards the satellite making it highly practical for mobile fleet such as trucks and offshore personnel.

With many advantageous characteristics as described, L-band is also being used for military purposes in a small portion $(1.3-1.7 \mathrm{GHz})$ allocated for satellite communication on Inmarsat. In search of a practical solution for efficient communication in combat zone and in general, military uses L-band satellite link to integrate all units in the battlefield to

Manuscript received May 12, 2015; revised August 13, 2015. Financial support for this research paper is provided by National Broadcasting and Telecommunications Commission, Bangkok, Thailand.

The authors are with National Broadcasting and Telecommunications Commission Bangkok, Thailand.

(e-mail: settapong.m@nbtc.go.th,_noppadol.t@nbtc.go.th, nattakit.s@nbtc.go.th) extend tactical information beyond line of sight, as an alternative to the very limited UHF satellite channels with only minor adaptations to the commonly used UHF systems [2].

In regards to antenna design, the shorter wavelength of L-band in comparison to UHF makes antenna much smaller and less expensive. Moreover, smaller antenna size allows military forces to conceal or disguise the operation when needed in the combat zone.

Among the popular choices for new generation of antenna technologies, microstrip patch antennas offer many attractive features such as low profile, light weight, easy fabrication, and intergrability with microwave circuits. Even though the design and implementation of wideband microstrip patch antenna can be a challenging task, many researchers still maintain their strong interest, especially for applications in L-band.

To cope with rising demand for modern portable wireless communication devices operating in multiple frequency bands and technologies, the proposed model is a dual-band circular microstrip patch antenna operating in L-Band for military applications.

\section{L-BAND APPLICATION AND TRENDS}

L-band is frequency range of $390 \mathrm{MHz}$ to $1.55 \mathrm{GHz}$. High frequencies usually suffer from signal loss when transported over copper coax cable like Intra-Facility Link. However, in the case of L Band, Low Noise Block (LNB) is adopted to convert high frequencies to $\mathrm{L}$ Band which can be transferred via Interfacility Link and processed by In-door-unit (IDU) telecommunication device. According to Plum Consulting (2012) L-band is widely available globally hence, allowing economies of scale. Therefore, L-band is the most cost effective solution for particularly satellite and terrestrial applications.

Currently, the L-band $1.4 \mathrm{GHz}$ is harmonized in Europe with frequency range between $1452 \mathrm{MHz}$ to $1492 \mathrm{MHz}$, with $40 \mathrm{MHz}$ of idle unpaired spectrum available. However, besides L-band $1.4 \mathrm{GHz}$, other countries have their own specific frequency range of L-band.

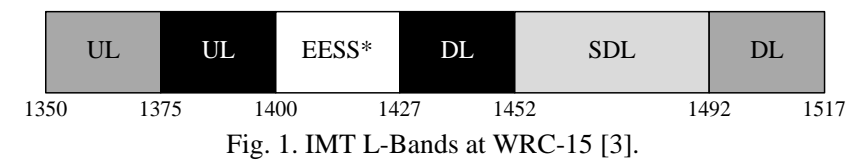

For mobile communications, L-band has attracted considerable attention from the industry during the past few years. Illustrated in Fig. 1, L-band was nominated as one of the most promising bands for IMT $(1350-1525 \mathrm{MHz}$ excluding $1400-1427 \mathrm{MHz}$ and $1695-1710 \mathrm{MHz}$ ) in the 
upcoming WRC-15 scheduled in November 2015, as part of the effort to respond to the rapidly increasing demand of mobile broadband. This medium frequency range is favorable for cellular communications because it offers a good balance between capacity and coverage, and penetrates buildings reasonably well [1]. Detailed in Fig.1, the trends for L-band are:

- Phase 1: 1452 - $1492 \mathrm{MHz}$ - Brazil is using this range for aeronautical mobile telemetry (AMT).

- $\quad$ Phase 2: 1375-1400 / 1427-1452 MHz (FDD, 2 × 25 $\mathrm{MHz}$ ). This range is used by fixed links and military, and is harmonized with CEPT (European Conference of Postal and Telecommunications Administrations). In Brazil, 1375 - $1400 \mathrm{MHz}$ is allocated to radio location and $1427-1452 \mathrm{MHz}$ is used by fixed links.

- Phase 3: $1350-1375 / 1492-1517$ (FDD, $2 \times 25$ ). The $1350-1375 \mathrm{MHz}$ is used by North Atlantic Treaty Organization (NATO). On the other hand, 1492-1517 was planned to be used for Wireless MICS by CEPT. In Brazil, 1350-1375 is allocated to radio location and 1492 $-1517 \mathrm{MHz}$ used by fixed links.

$\mathrm{L}$ band is a key to identify new harmonized spectrum for IMT in WRC-15. Further, this research seeks to provide a proposed dual-band circular microstrip patch antenna operating in L-Band for military applications for bandwidth $1.62-1.7 \mathrm{GHz}$ and $2.74-2.8 \mathrm{GHz}$.

\section{ANTENNA DESIGN}

In addition to compact size of the antenna, operation at two or more discrete frequency bands is an important feature. This kind of design avoids the use of multiple antennas, like for instance, by integrating, receiving and transmitting functions into the same communication system or the same antenna operating in the L-band applications. In this paper, the proposed antenna is given in Fig. 2. It has a substrate of permittivity $\varepsilon_{r}$, radius $(a)$ and thickness $h$. Inset feeding is used as a feeding method.

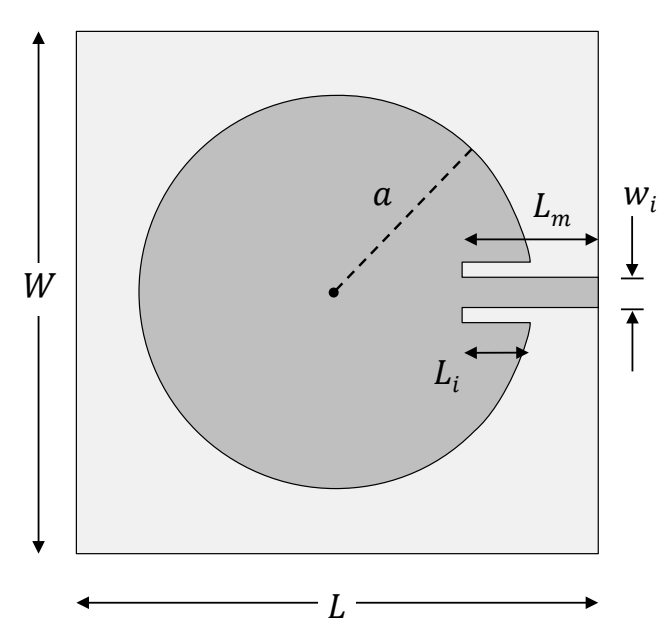

Fig. 2. Geometry of the microstrip patch antenna.

Then, the proposed antenna that operates at the specified operating frequency $f_{r}$.

Formulas used for calculating

$$
a=F\left\{1+\frac{2 h}{\pi F \varepsilon_{r}}\left[\ln \left(\frac{\pi F}{2 h}\right)+1.7726\right]\right\}^{-1 / 2}
$$

where,

$$
F=\frac{8.791 \times 10^{9}}{f_{r} \sqrt{\varepsilon_{r}}} ; f_{r} \text { in GHz, h in } \mathrm{mm} .
$$

The frequency-dependent Smith-Chart model (FDSC) is used to decrease error on frequency-dependent characteristics [4]. Based on previous research studies [5]-[7], FDSC increases result accuracy in models adopted in past literature [8].

To achieve frequency-dependent characteristic impedance $\left(Z_{0}{ }^{\prime}(\omega)\right)$, the frequency-dependent capacitance $(C(\omega))$ derived in [9] is replaced into the capacitance $(C)$ in the formulation of $Z_{0}=\sqrt{L / C}$. Therefore, frequency-dependent characteristic impedance is

$$
Z_{0}^{\prime}(\omega)=\sqrt{\frac{L}{C[A(\omega)-j B(\omega)]}}=\frac{Z_{0}}{\sqrt{A(\omega)-j B(\omega)}}
$$

Further frequency-dependent (lossy) Smith-chart is derived from $Z_{0}^{\prime}(\omega)$ in Eqn. (3) into the normalized terminal impedance expression as done in traditional Smith-chart model [10], [11]. Therefore the normalized terminal impedance $Z_{L}^{\prime}$ is

$$
Z_{L}^{\prime}=\frac{Z_{L}}{z_{0}^{\prime}(\omega)}=b r+j b x \quad(\text { Dimensionless })
$$

As $r$ and $x$ are the normalized resistance and normalized reactance, , and $b=\sqrt{A(\omega)-j b(\omega)}$ [11].

The voltage reflection coefficient of present Smith chart is

$$
\Gamma^{\prime}=\Gamma_{r}^{\prime}+j \Gamma_{i}^{\prime}=\frac{z_{L}^{\prime}-1}{z_{L}^{\prime}+1}
$$

or

$$
Z_{L}^{\prime}=\frac{Z_{L}}{Z_{0}^{\prime}(\omega)}=b r+j b x=\frac{\left(1+\Gamma_{r}^{\prime}\right)+j \Gamma_{i}^{\prime}}{\left(1-\Gamma_{r}^{\prime}\right)-j \Gamma_{i}^{\prime}}
$$

Further the set of equations that represent the modified Smith-chart are: [11]

$$
\left(\Gamma_{r}^{\prime}-\frac{b r}{1+b r}\right)^{2}+{\Gamma_{i}^{\prime}}^{2}=\frac{1}{(1+b r)^{2}}
$$

and

$$
\left(\Gamma_{r}^{\prime}-1\right)^{2}+\left(\Gamma_{i}^{\prime}-\frac{1}{b x}\right)^{2}=\left(\frac{1}{b x}\right)^{2}
$$

IV. Simulation Results

TABLE I: ANTENNA DESIGN PARAMETERS

\begin{tabular}{ccc}
\hline \hline Parameters & \multicolumn{2}{c}{ Value } \\
\hline$\varepsilon_{r}$ & 4.4 & \\
$h$ & 1.7 & $\mathrm{~mm}$ \\
$L_{i}$ & 5.2 & $\mathrm{~mm}$ \\
$W_{i}$ & 1.2 & $\mathrm{~mm}$ \\
$L_{m}$ & 25.5 & $\mathrm{~mm}$ \\
$a$ & 25.3 & $\mathrm{~mm}$ \\
\hline \hline
\end{tabular}

The present design of the proposed antenna uses FR4 dielectric as a substrate. The size of the ground plate and substrate is $100 \times 100 \mathrm{~mm}^{2}$. The essential parameters for 
the design are given in Table I.

Based on the simulation results by using lE3D software and MATLAB programming, the proposed antenna resonates at $1.66 \mathrm{GHz}$ with $-22.7 \mathrm{~dB}$ return loss and at $2.77 \mathrm{GHz}$ with $-15.5 \mathrm{~dB}$ return loss. Indicated in the Fig. 3 , the bandwidth of frequency at $1.66 \mathrm{GHz}$ and $2.77 \mathrm{GHz}$ is $80 \mathrm{MHz}$ and $60 \mathrm{MHz}$, respectively.

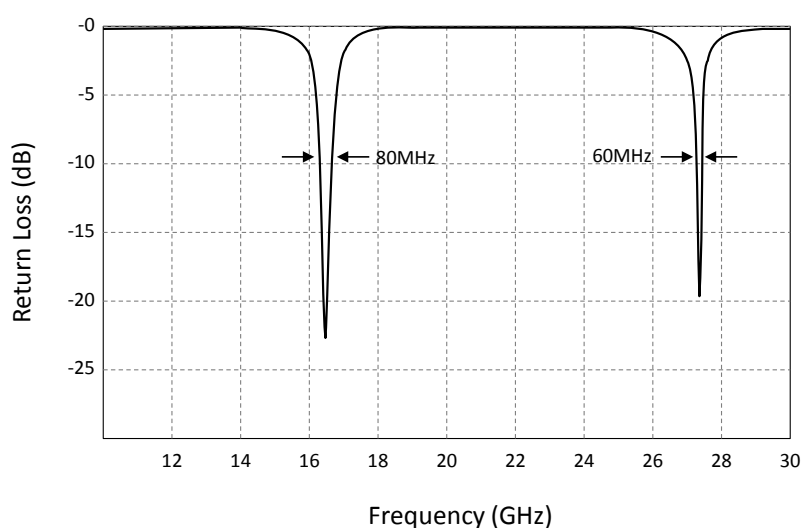

Fig. 3. The bandwidth of frequency at $1.66 \mathrm{GHz}$ and $2.77 \mathrm{GHz}$ is $80 \mathrm{MHz}$ and $60 \mathrm{MHz}$.

\section{FUTURE WORK}

For the future research, we will propose a model to analyze the performance of a circular microstrip antenna. The theory for this proposed model is addressed from [12].

To achieve this goal, an approach that uses the Debye relation [13] is presented to illustrate the frequency-dependent characterization of a microstrip structure. Moreover, "a reactive relaxation diagram” (analogous to the Cole-Cole diagram) shows the frequency-dependent capacitive effects and associated loss in a circular microstrip structure. This model contributes to computer-aided microstrip circuit designs.

\section{A. Dielectric Permittivity}

Dielectric permittivity $(\varepsilon)$ is ratio of the electric field $E_{0}$ in free-space to that is, material $E$ for the same distribution of charge. This quantity is a measure in capacitors, which consists of parallel plates of area $A$ separated by a distance $d$, and is small in comparison with $A$.

Therefore, if charges on the plates remain the same then the space between them is filled with a dielectric material so, the capacitance $C$ of the condenser is

$$
C=A \varepsilon / d
$$

where $\varepsilon=\varepsilon_{0} \varepsilon_{r}$ and $\varepsilon_{0}=(1 / 36 \pi) \times 10^{-9} \mathrm{~F} / \mathrm{m}$ denotes the free-space permittivity.

\section{B. Dielectric Relaxation Process}

The term relaxation is defined as "time-dependent return to equilibrium of a system that has been perturbed by a change in an applied constraint" [12]. When the constraint is the electric field as in a capacitor, the observable quantity that varies with time is the dielectric polarization, giving rise to a dielectric relaxation.

The relaxation process is vital for interaction between field and dielectric. The Debeye model depicts materials in terms of individual dipole moments which has variable charge separation in a viscous medium. A dipole is subjected to an electric field and aligns and elongate in direction of applied electric field in finite time. However, if externally applied field is discontinued all the torques due to the external field on the dipoles vanishes instantly, and, the dipole "relaxes" to the initial state as an exponential decay process [12].

The constant electric field $\mathrm{E}$ to a dielectric resolves into the development of an electric displacement $\mathrm{D}$ that increases with time until a constant value is reached if time is sufficiently long. After removal of the electric field, the electric displacement returns with time to zero [12].

Small electric displacements, $\varepsilon=|D| /|E|$; is such that the system is linear, and, consequently, it conforms to the superposition principle [13]. The dielectric constant is a time-dependent property, its value creasing from $\varepsilon_{\infty}$ for $t=0$ to $\varepsilon_{s}$ for $t \rightarrow \infty$. The electric displacement can obviously be written as

$$
D(t)=\left[\varepsilon_{\infty}+\left(\varepsilon_{s}-\varepsilon_{\infty}\right) \Phi(t)\right] E
$$

where $\Phi(t)$ is the built-up normalized dielectric function whose extreme values are

$$
\begin{array}{ll}
\Phi(t)=1-\phi(t)=0 & \text { for } t=0 \\
\Phi(t)=1-\phi(t)=1 & \text { for } t=\infty
\end{array}
$$

where $\phi(t)$, the normalized decay function of the polarization when a steady macroscopic electric field is removed from the medium, acquires the values 1 and 0 at $t=0$ and $t=\infty$, respectively. If an electric field is applied at $\mathrm{t}=0$, it increase by infinitesimal amount, $\mathrm{dE}$, at $t=$ $\theta(0<\theta<t)$, such that the increase is

$$
d D=\varepsilon_{\infty} d E+\left(\varepsilon_{s}-\varepsilon_{\infty}\right) \Phi(t=\theta) d E
$$

In linear system, the total displacement at time $t$ caused by a variable electric field $E(\theta)$ is the result of the superposition of all the increments, $d D$ and

$$
D(t)=\varepsilon_{\infty} E(t)+\left(\varepsilon_{s}-\varepsilon_{\infty}\right) \int_{-\infty}^{t} \frac{d E(\theta)}{d \theta} \Phi(t-\theta) d \theta
$$

By making the substitution $(t-\theta)=\xi$ and integrating by parts, one finds

$$
D(t)=\varepsilon_{\infty} E(t)+\left(\varepsilon_{s}-\varepsilon_{\infty}\right) \int_{0}^{\infty} \frac{d \Phi(\xi)}{d \xi} E(t-\xi) d \xi
$$

For an alternating electric field, $E=E_{0} \exp (j \omega t)$, Eqn.(15) leads to

$$
\varepsilon_{m}^{*}(\omega)=D(t) / E(t)=\varepsilon_{\infty}+\left(\varepsilon_{s}-\varepsilon_{\infty}\right) \int_{0}^{\infty} \exp (-j \omega \xi)\left[-\frac{d \phi(\xi)}{d \xi}\right] d \xi
$$

where $-d \phi(\xi) / d \xi=d \phi(\xi) / d \xi$, and $\varepsilon_{\mathrm{m}}^{*}(\omega)=\varepsilon_{\mathrm{m}}^{\prime}(\omega)-$ $j \varepsilon_{m}^{\prime \prime}(\omega)$ is the complex dielectric permittivity. Rearrangement of the terms of Eqn.(16) gives [13].

$$
\frac{\varepsilon_{m}^{*}(\omega)-\varepsilon_{\infty}}{\varepsilon_{S}-\varepsilon_{\infty}}=\int_{0}^{\infty}(-d \phi / d t) \exp (-j \omega t) d t
$$


The solution of the integral involves the decay function over relaxation range. For a good relaxation range, the rate of return to the equilibrium, $P_{e q}$, and, as a first approximation, the phenomenon is depicted by linear first-order approximation with the solution $P-P_{e q} \approx \exp \left(-t / \tau_{r}\right)$. Parameter $\tau_{r}$ is called the relaxation time, which may be defined as the time in which the observable quantity is reduced to $1 / e$ times its original value [12]. For a dielectric relaxation involving a single relaxation time (Debye relaxation), $\phi(t) \approx \exp \left(-t / \tau_{r}\right)$ and Eqn. (17) becomes

$$
\begin{gathered}
\frac{\varepsilon_{m}^{*}(\omega)-\varepsilon_{\infty}}{\varepsilon_{S}-\varepsilon_{\infty}}=\frac{1}{1+j \omega \tau_{r}} \\
\varepsilon_{m}^{*}(\omega)=\varepsilon_{\infty}+\frac{\varepsilon_{S}-\varepsilon_{\infty}}{1+j 2 \pi\left(\omega / \omega_{r}\right)}
\end{gathered}
$$

where $\varepsilon_{m}^{*}(\omega)$ is the complex permittivity of the dielectric material; $\varepsilon_{\infty}$ is the permittivity at infinite frequency; $\varepsilon_{\mathrm{s}}$ is the static or d.c. permittivity; $\omega=2 \pi f$; $f$ is the applied frequency; and $\tau_{\mathrm{r}}\left(1 / \mathrm{f}_{\mathrm{r}}\right)$ is the relaxation time of the dielectric material and it can be decomposed as:

$$
\varepsilon_{m}^{*}(\omega)=\left[\varepsilon_{\infty}+\frac{\varepsilon_{s}-\varepsilon_{\infty}}{1+4 \pi^{2}\left(\omega / \omega_{r}\right)^{2}}\right]-j\left[\left(\varepsilon_{s}-\varepsilon_{\infty}\right) \frac{2 \pi\left(\omega / \omega_{r}\right)}{1+4 \pi^{2}\left(\omega / \omega_{r}\right)^{2}}\right]
$$

Therefore, the real $\varepsilon_{m}^{\prime}(\omega)$ and loss $\varepsilon_{m}^{\prime \prime}(\omega)$ components of $\varepsilon_{\mathrm{m}}^{*}(\omega)$ are given by

$$
\begin{gathered}
\varepsilon_{m}^{\prime}(\omega)=\left[\varepsilon_{\infty}+\frac{\varepsilon_{s}-\varepsilon_{\infty}}{1+4 \pi^{2}\left(\omega / \omega_{r}\right)^{2}}\right] \\
\varepsilon_{m}^{\prime \prime}(\omega)=\left[\left(\varepsilon_{s}-\varepsilon_{\infty}\right) \frac{2 \pi\left(\omega / \omega_{r}\right)}{1+4 \pi^{2}\left(\omega / \omega_{r}\right)^{2}}\right]
\end{gathered}
$$

Eqn. (21) and (22) are usually called Debye equations. The functions represented by Eqn. (21) and (22) are illustrated in Fig. 4.

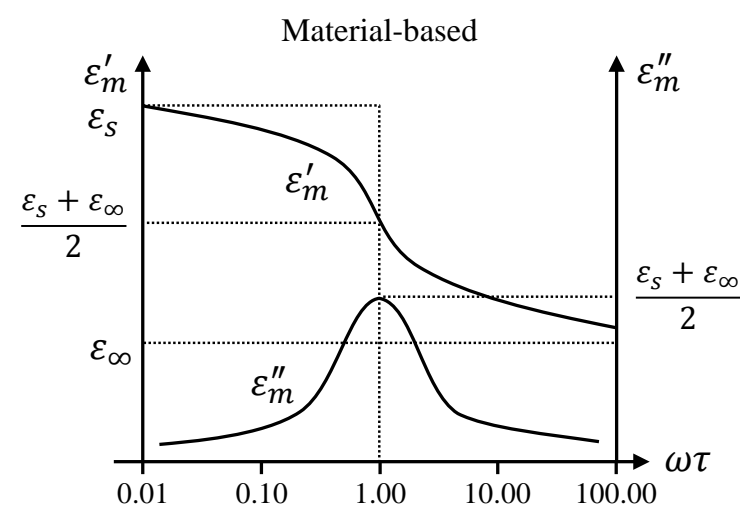

Fig. 4. Dielectric relaxation: Debye relation.

Experimentally, one measures usually the quantities $\varepsilon_{m}^{\prime}(\omega)$ and the loss tangent [14]

$$
\tan \left(\delta_{d}\right)=\frac{\varepsilon^{\prime \prime}(\omega)}{\varepsilon^{\prime}(\omega)}
$$

and it is convenient to know the Debye equations in terms of the loss tangent, when

$$
\tan \left(\delta_{d}\right)=\frac{\left(\varepsilon_{S}-\varepsilon_{\infty}\right) \omega \tau_{r}}{\varepsilon_{S}+\varepsilon_{\infty} \omega^{2} \tau_{r}^{2}}
$$

In the limit of low and high frequencies, Eqn. (21) and (22) lead to

$$
\lim _{\omega \rightarrow 0} \varepsilon_{m}^{\prime}(\omega)=\varepsilon_{s}
$$

$$
\lim _{\omega \rightarrow \infty} \varepsilon_{m}^{\prime}(\omega)=\varepsilon_{\infty}
$$

Eqn. (21) and (22) imply $\varepsilon^{\prime}(\omega)$ is a decreasing function of frequency, $\varepsilon^{\prime \prime}(\omega)$ such that it approaches zero for small and for large values of frequency, reaching a maximum at $\omega \tau_{r}=1$. Hence, relaxation time is equivalent to reciprocal of the angular frequency at the maximum of the loss absorption. Debye relation is derived from these functions:

1) Local field at any point in the medium is the same as the applied field.

2) D.C. conductivity of the material is negligible.

3) All the dipoles have identical relaxation time $\tau_{r}$.

The real and imaginary parts of the Debye relation are depicted in Fig. 4. The Debye relation can be represented as shown in Fig. 5. It is known as the Cole-Cole diagrams. This graphical representation which is of considerable practical importance involves plotting $\varepsilon^{\prime}$ versus $\varepsilon^{\prime \prime}$. The function $\varepsilon^{\prime \prime}\left(\varepsilon^{\prime}\right)$ can be obtained by elimination of $\omega$ between the equations $\varepsilon_{m}^{\prime}(\omega)$ and $\varepsilon_{m}^{\prime \prime}(\omega)$. The functional relation obtained in this format is depicted as a circle. $\varepsilon^{*}$ is now presented by the semi-circle of radius $\left(\varepsilon_{\mathrm{s}}-\varepsilon_{\infty}\right) / 2$ centered at $\varepsilon^{\prime}=\left(\varepsilon_{\infty}+\varepsilon_{\mathrm{s}}\right) / 2$. The top of this semi-circle corresponds to $\omega \tau_{\mathrm{r}}=1$. The relaxation time is calculated from $\tau_{r}=1 / f_{r}$. It can be seen that $\varepsilon^{\prime}$ decreases with frequency. This means that the energy $\varepsilon^{\prime}|E|^{2} / 2$ stored in the material will decrease with frequency.

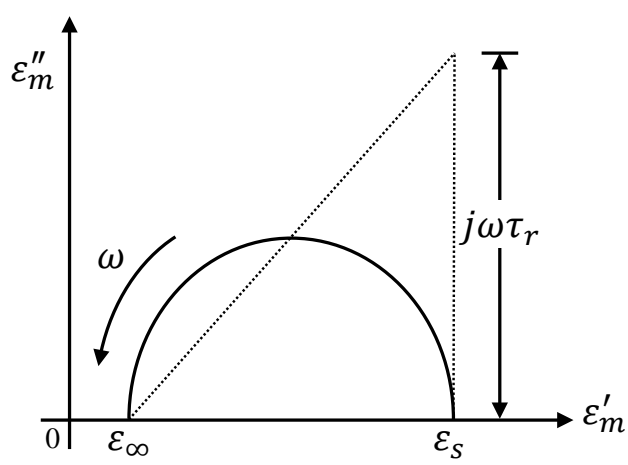

Fig. 5. Cole-Cole diagram of material-based.

From the form of the asymptotic lower bound derived by the [15], we concludes that an asymptotic approximation to the capacitance when $d / a \rightarrow 0$ is of the form

$$
C \simeq \frac{a^{2} \pi \epsilon_{r} \epsilon_{0}}{d}\left\{1+\frac{2 d}{\pi \epsilon_{r} a}\left[\ln \left(\frac{a}{2 a}\right)+g\left(\epsilon_{r}, \frac{d}{a}\right)\right\}+o(1)\right.
$$

An analytic expression for the function $g\left(\epsilon_{r}, d / a\right)$ is difficult to derive. We also note that the asymptotic lower bound of the dielectric case differs from the free space case by a term

$$
-\frac{4 \epsilon_{r}^{3}}{1-\epsilon_{r}^{2}} \sum_{n=2}^{\infty} n^{2} \ln n\left(\frac{1-\epsilon_{r}}{1+\epsilon_{r}}\right)^{n}
$$

which can be shown to be almost a linear function in $\epsilon_{r}$ (Fig. 6).

We will use all of an aforementioned result to propose a model to analyze performance of the circular microstrip antenna for the future research. 


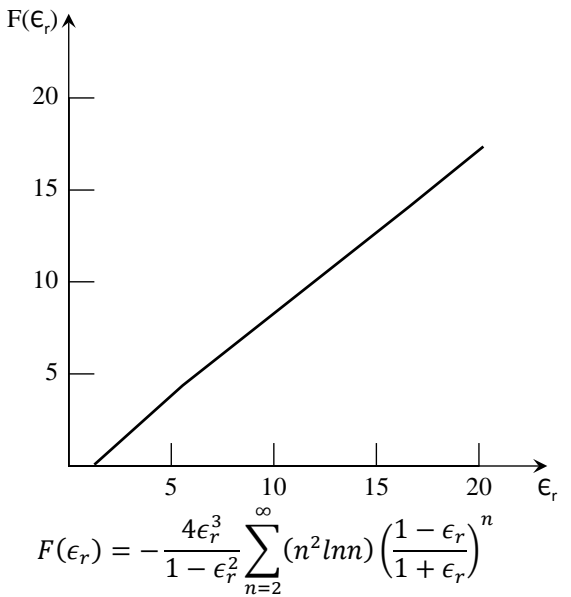

Fig. 6. The function $F\left(\epsilon_{r}\right)$.

\section{CONCLUSION}

In this research a representation of dual-band circular patch microstrip antenna for L-band military applications for bandwidth $1.62-1.7 \mathrm{GHz}$ and $2.74-2.8 \mathrm{GHz}$ is proposed. The design of the proposed antenna features FR4 dielectric as a substrate. The simulation results from IE3D software and MATLAB programming shows that the proposed antenna resonates at $1.66 \mathrm{GHz}$ with $-22.7 \mathrm{~dB}$ return loss and at $2.77 \mathrm{GHz}$ with $-15.5 \mathrm{~dB}$ return loss. In conclusion, the bandwidth of the frequency at $1.66 \mathrm{GHz}$ and $2.77 \mathrm{GHz}$ is 80 $\mathrm{MHz}$ and $60 \mathrm{MHz}$. The paper also discussed about the direction of the future work.

\section{REFERENCES}

[1] GSMA, "GSMA public policy position: mobile spectrum requirements and target bands for WRC," 2015.

[2] Immarsat. (2014). Beyond Line of Sight Communications: Using Existing Military Radios. [Online]. Available: https://www.inmarsatgov.com/uploadedFiles/Resources/Downloads/ltac-whitepaper.pdf

[3] ITU and Qualcomm. (2014). L Band: Harmonization Opportunity in Latam. [Online]. Available: http://www.itu.int/en/ITU-D/Regional-Presence/Americas/Documents /EVENTS/2014/0819-PA-IMT/2nd\%20CRT\%20-\%20L\%20band\%2 Oharmonization\%20-\%20Geraldo\%20Neto\%2019.08.14\%20sem\%20 notes.pdf

[4] R. Garg, Microstrip Antenna Design Handbook, Artech House, 2001.

[5] S. Malisuwan and M. Charoenwattanaporn, "Modified Smith-chart representation as applied to microstrip antenna design for wireless LAN applications," in Proc. the 2003 IEEE International Symposium on Electromagnetic Compatibility, 2003, pp. 150-153, vol.1.

[6] S. Malisuwan, P. Neelakanta, and V. Ungvichian, "A Cole-Cole diagram representation of microstrip structure," Applied Computational Electromagnetics Society Journal, vol. 15, pp. 167-174, 2000.

[7] P. S. Neelakanta, Handbook of Electromagnetic Materials: Monolithic and Composite Versions and Their Applications, CRC press, 1995

[8] F. Abboud, J. P. Damiano, and A. Papiernik, "Simple model for the input impedance of coax-fed rectangular microstrip patch antenna for
CAD,” Microwaves, Antennas and Propagation, IEEE Proceedings $H$, vol. 135, pp. 323-326, 1988.

[9] S. Malisuwan, M. Charoenwattanapom, S. Huvanandana, and K. Rosesukon, "Design of microstrip antenna for Bluetooth and WLAN applications by applying modified Smith-chart representation," in Proc. the 2003. Asia-Pacific Conference on Applied Electromagnetics, APACE, 2003, pp. 38-41.

[10] J. C. Freeman, Fundamentals of Microwave Transmission Lines, Wiley, 1996.

[11] S. Malisuwan and J. Sivaraks, "Frequency-dependent smith-chart model as applied to integrated circuit package antenna design," International Journal of Computer and Communication Engineering, vol. 2, no. 5, pp. 625-629, 2013, 2013.

[12] S. Malisuwan, J. Sivaraks, and N. Suriyakrai, "The performance of rectangular microstrip structure by applying the cole-cole diagram concept,” Lecture Notes on Software Engineering, vol. 2, p. 48, 2014.

[13] E. Riande and E. Saiz, Dipole Moments and Birefringence of Polymers, Prentice Hall, 1992.

[14] V. V. Daniel, Dielectric Relaxation, Academic Press, 1967.

[15] W. C. Chew and J. A. Kong, "Effects of fringing fields on the capacitance of circular microstrip disk," IEEE Transactions on Microwave Theory and Techniques, vol. 28, pp. 98-104, 1980.

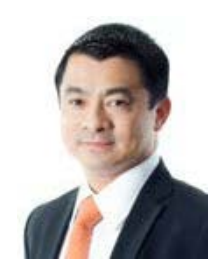

Settapong Malisuwan was born on March 24, 1966 in Bangkok, Thailand. He received his $\mathrm{PhD}$ in electrical engineering (telecommunications), specializing in mobile communication systems from Florida Atlantic University (State University System of Florida), Boca Raton in 2000. He received his MSc in electrical engineering in mobile communications system, from George Washington University in 1996, and MSc in electrical engineering in telecommunication engineering from Georgia Institute of Technology in 1992. He served in the Royal Thai Armed Forces for more than 25 years and is currently the vice chairman of National Broadcasting and Telecommunications and the chairman of Telecommunication Commission Bangkok, Thailand. His research interests are in electromagnetics, efficient spectrum management and Telecommunications policy and management.

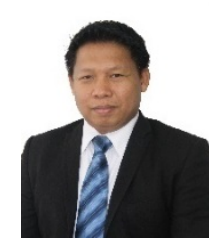

Noppadol Tiamnara was born on November 12, 1968 in Pah Na Korn Sri Ayuttaya, Thailand. He received his BSc in electrical engineering from Saint John's University, Thailand, 2002. He received his MSc in technology management from thammasart university, Thailand, 2012. Since 2006, he has been working in National Broadcasting and Telecommunications Commission as assistant to secretary of vice chairman of National Broadcasting and Telecommunication Commission (NBTC). His research interests include LTE design, wireless systems, microstrip antenna and applied electromagnetic.

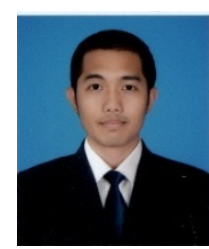

Nattakit Suriyakrai was born in Khonkhaen, Thailand on March 22, 1987. He received his bachelor of liberal arts in Japanese Language from Thammasat University in 2010. He has been working as an Assistant to the vice chairman in National Broadcasting and Telecommunications, Bangkok, Thailand since November 2012. His research interests are in technology management and spectrum management. 